\title{
A SECURE STRUCTURE FOR HIDING INFORMATION IN A CRYPTOSYSTEM BASED ON MACHINE-LEARNING TECHNIQUES AND CONTENT-BASED OPTIMIZATION USING PORTFOLIO SELECTION DATA
}

\author{
CHANCHAL KUMAR*AND MOHAMMAD NAJMUD DOJA †
}

\begin{abstract}
Many systems including networks environment have higher complexity and ubiquitous connections than a normal system, hence design of a security system for hiding pertinent data a challenging task. This paper presents a secure structure that extends a protocol which is available for fast Diffe-Hellman protocol using Kummer surface. We show the extended version of the scheme by inclusion of an additional point, such that a more secure system can be constructed. The scheme has been build by employing machine-learning technique to select an appropriate class from multiple set of surfaces. A brief discussion on inclusion of multiple surfaces and making a selection of a specific surface using NSGA II algorithm is also provided. In this paper, we provide a brief overview of AES-128 (AES also known as Rijndael). In the starting, a short overview of the AES is given. This paper also has a description for altering the key generation module in AES based upon a newly designed content-based matrix which is built from portfolio selection data. The matrix is constructed using some predefined factors modifies the existing index which is computed based upon the context of the message. An optimization algorithm is employed for selecting specified entries from content matrix. These selected entries are used for altering the key generation algorithm in AES. The modified output obtained after altering the key generation scheme is provided in the paper. Lastly, a brief overview of LIM index, which is used as an index in cryptanalysis, is given. This paper has a description of the scheme to construct a more secure system that is capable of hiding the information with above-mentioned techniques.
\end{abstract}

Key words: Deffie-Hellman key exchange, Machine Learning techniques, NSGA II (Non-dominated Sorting Genetic Algorithm II), Key generation (AES) content based matrix, Optimization, LIM Index

AMS subject classifications. $68 \mathrm{M} 12$

1. Introduction. The main objective of a cryptographic system may be stated as providing a capability for secure information exchange over a network. The plain text is the body of the message. Encryption is achieved by coding the input message using an algorithm along with a secret key. The coded message is called cipher-text. On the receiving side, the message is decoded using the secret key and reversing the steps of encryption algorithm. Thus, the plain text is obtained using the same secret key and decryption algorithm. The information which is encoded yields different sets of cipher. The study of information about structure of the cipher could be very beneficial for analysis. Cryptology deals with performing cryptanalysis in order to challenge the security of the encryption algorithm. Diffie-Hellman protocol is a commonly used protocol for secretly exchanging key [1]. In this protocol, the sender and receiver take a decision to select a finite set of points (Group) A, and choose a generator (v) from a subgroup. Using the private key $\left(x_{1}\right)$ of the sender, a secret number $n_{1}=\left(\alpha^{x_{1}} \bmod p\right)$ is generated. Here, $\mathrm{p}$ is a prime number and $\alpha$ is a primitive-root of $\mathrm{p}$ such that $\alpha<p$. The receiver generates a secret number $n_{2}=\left(\alpha^{x_{2}} \bmod p\right)$ where $x_{2}$ is the private key of the receiver.The secret key exchange can be achieved by

$$
\begin{aligned}
& k_{1}=n_{2}^{x_{1}} \bmod p \\
& k_{2}=n_{1}^{x_{2}} \bmod p
\end{aligned}
$$

Recently, a fast version of this protocol is provided that makes use of Kummer Surface [1, 23, 24]. In this paper, a new scheme is proposed for enhancing the security of the protocol by adding another point $\left(u_{5}\right)$ on the surface. A brief overview of a scheme that can be incorporated for selecting one of the multiple surfaces using NSGA II is given in the paper.

1.1. A Brief Overview of AES. During 1990's, it became clear that DES (Data Encryption Standard) will not be able to meet the required security standard [2]. In the year 1997, AES was adopted by NIST. AES was selected among other available ciphers. AES was chosen as a replacement for DES. AES now has a bigger key size and it is capable of handling the type of attacks meant for DES. In 2001, AES Rijndael was chosen

*Department of Computer Engineering, Jamia Millia Islamia, India (kumarchanchal943@gmail.com)

${ }^{\dagger}$ Department of Computer Engineering, Jamia Millia Islamia, India (ndoja@yahoo.com) 
among several other variants of ciphers. Previously DES was attacked using linear and differential cryptanalysis, these attacks were not successful in case of AES. AES uses block structure that means plain text is processed as blocks and the encryption is achieved on a block structure. AES composed of algebraic blocks which are relatively simple.

1.2. Different stages used in AES. AES is based upon block structure. The input plain text is divided in to various blocks of fixed size. The encryption takes place with each block separately. The size of the block is 128 bits. The allowed key size could be 128,192 or 256 bits.

AES works with Galois Field (GF) $\left(2^{8}\right)$, a polynomial $\left(b_{7} x^{7}+b_{6} x^{6}+\ldots . b_{0}\right)$

is utilized for representing 8 bits (byte). Hexadecimal notation is used to represent bytes.

State: We are considering key size of 128 bits. The results are represented as state which is 128 bits in length and different operations are performed on the state. A matrix consisting of 4 rows and 4 columns is used as given below:

$$
\left[\begin{array}{llll}
x(0,0) & x(0,1) & x(0,2) & x(0,3) \\
x(1,0) & x(1,1) & x(1,2) & x(1,3) \\
x(2,0) & x(2,1) & x(2,2) & x(2,3) \\
x(3,0) & x(3,1) & x(3,2) & x(3,3)
\end{array}\right]
$$

The state is opterated with multiple rounds. Each round contains substitution box, Diffusion operation (Linear) and xoring with round key generated for each round.

The Diffusion operation consist of performing the shift rows operation then performing the transformation which is centred around mixing the different columns. For generating the round key for each round, the original key is used to generate a sub key for each round. Lastly, the algorithm used in Rijndael may described as below:

Firstly, the input message is placed in a state of $4 \times 4$ matrix. This state is xored with round key of $0^{\text {th }}$ round. Next, ten rounds are applied where the last round skips the mix column operation. The final state is the desired ciphertext.

This paper has description of a scheme that alters the key generation algorithm using a content-based matrix. The output of different keys for each round is given. The alteration in key generation is intended to enhance the hiding capability of AES and making it secure against different kinds of attacks.

1.3. Related Work. The use of theories of fuzzy extractor and secure sketch in the framework of key generation from biometrics is proposed in [4]. A description of lossy trapdoor function with their applications is given in $[5,6]$. A significant use of pseudo-random number generators is presented in [7, 8]. A public key cryptosystem is more adaptive and secure against adaptive chosen ciphertext attack [9]. LIM (Lorenz Information Measure) based Cryptanalysis of AES-128 and AES-256 Block is presented in [11]. Some mathematical attacks like differential and linear cryptanalysis decrease the key search space but they not able to break the AES [12]. A description of some versions of Even-Mansour cipher scheme is given in [13]. Randomization based AES is better than first order differential electromagnetic and power analyses in term of low execution cost [14]. A combined approach of image processing and laser fault injections is given in [15] for security characterization of a hardware AES. A complete solution based on key updating framework to secure the execution of any kind of AES operation is proposed in [16]. A systematic toolbox is proposed in [17] for white-box implementation. A general study of the relationship between cryptography and machine learning is given in [18]. The primal machine algorithms are well performed in order to construct the encrypted models [19, 20, 21]. To perform successful key recovery deep learning techniques are appropriate [22]. A significant use of Kummer Surfaces is provided in $[23,24]$. Symmetric and asymmetric cryptographic techniques based a new security protocol is proposed in [27] for online transaction.

2. Proposed System. One of the vastly used algorithm for secure key exchange is Diffie-Hellman protocol. Kummer surface is recently proposed protocol for a fast-version of Diffie-Hellman protocol. This section provides description of the new protocol build using an additional point $\left(u_{5}\right)$ in the extended surface. The details of the protocol are given below: 


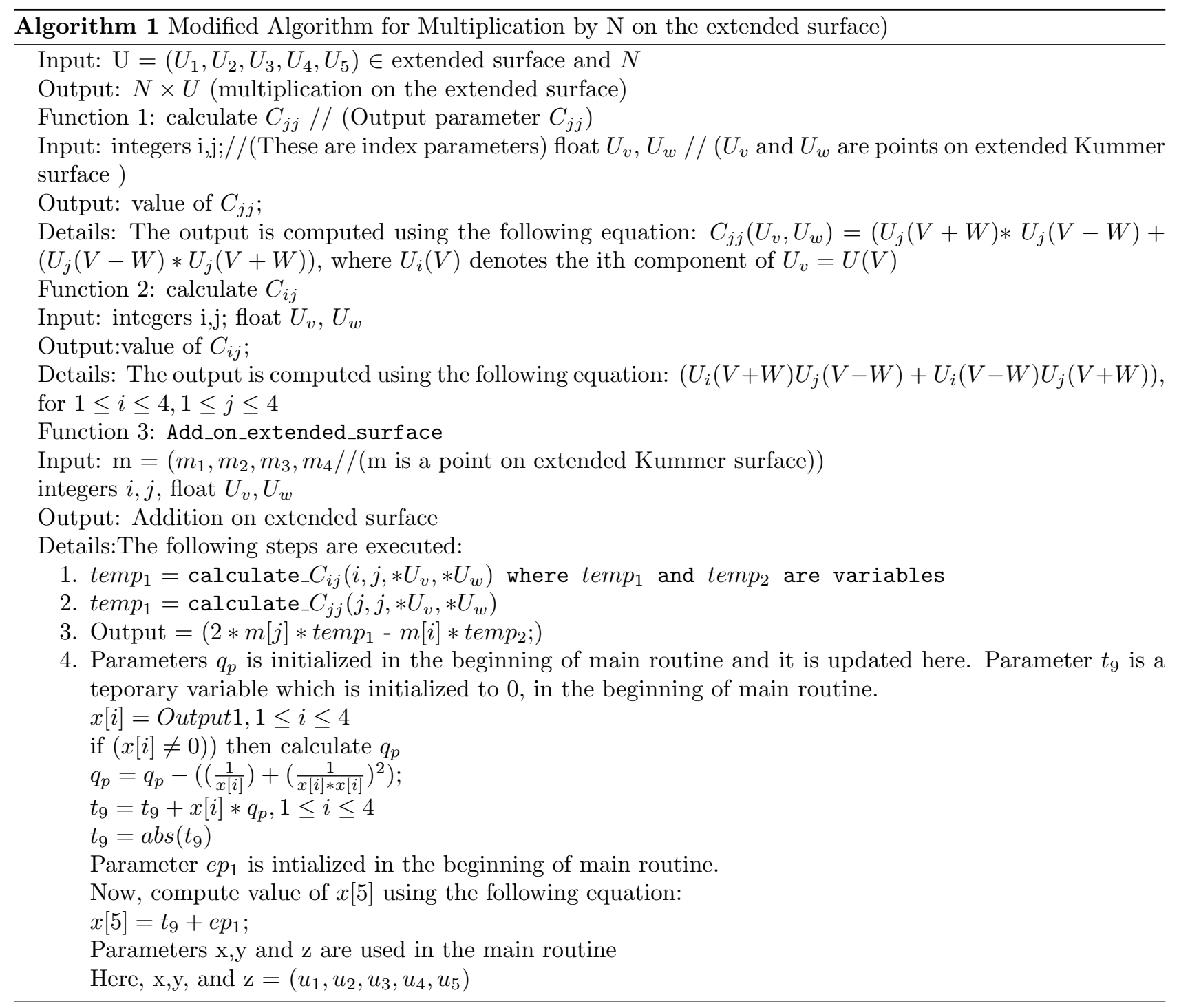

2.1. New Scheme adopted for inclusion of point $\left(u_{5}\right)$ in the extended surface. The description of new method based on inclusion of point $\left(u_{5}\right)$ in extended surface is presented here:

Need: A variant of Diffie-Hellman protocol based on Kummer surface is described in [5]. To enhance the security aspects of the structure, a new point $\left(u_{5}\right)$ is being added. A sample run with of $\left(u_{1}, u_{2}, u_{3}, u_{4}, u_{5}\right)$ is provided in the paper.

Significance: The additional point $\left(u_{5}\right)$ could prove a quite useful index for decision-making. The computed values of $\left(u_{1}, u_{2}, u_{3}, u_{4}, u_{5}\right)$ are used in a machine-learning algorithm to select a specific surface, in case, the design considers multiple surfaces and then chooses one surface for multiplication.

Impact: The selected surface based on computed value of $\left(u_{5}\right)$ will be utilized to perform a multiplication. The output obtained with added point $\left(u_{1}, u_{2}, u_{3}, u_{4}, u_{5}\right)$ are given below. The details of main routine along with various functions used are given below:

2.1.1. Algorithm for Machine-learning for a sample data set of three extended surfaces. A sample technique for classification based on Machine-learning is described below given in algorithm 3 . 


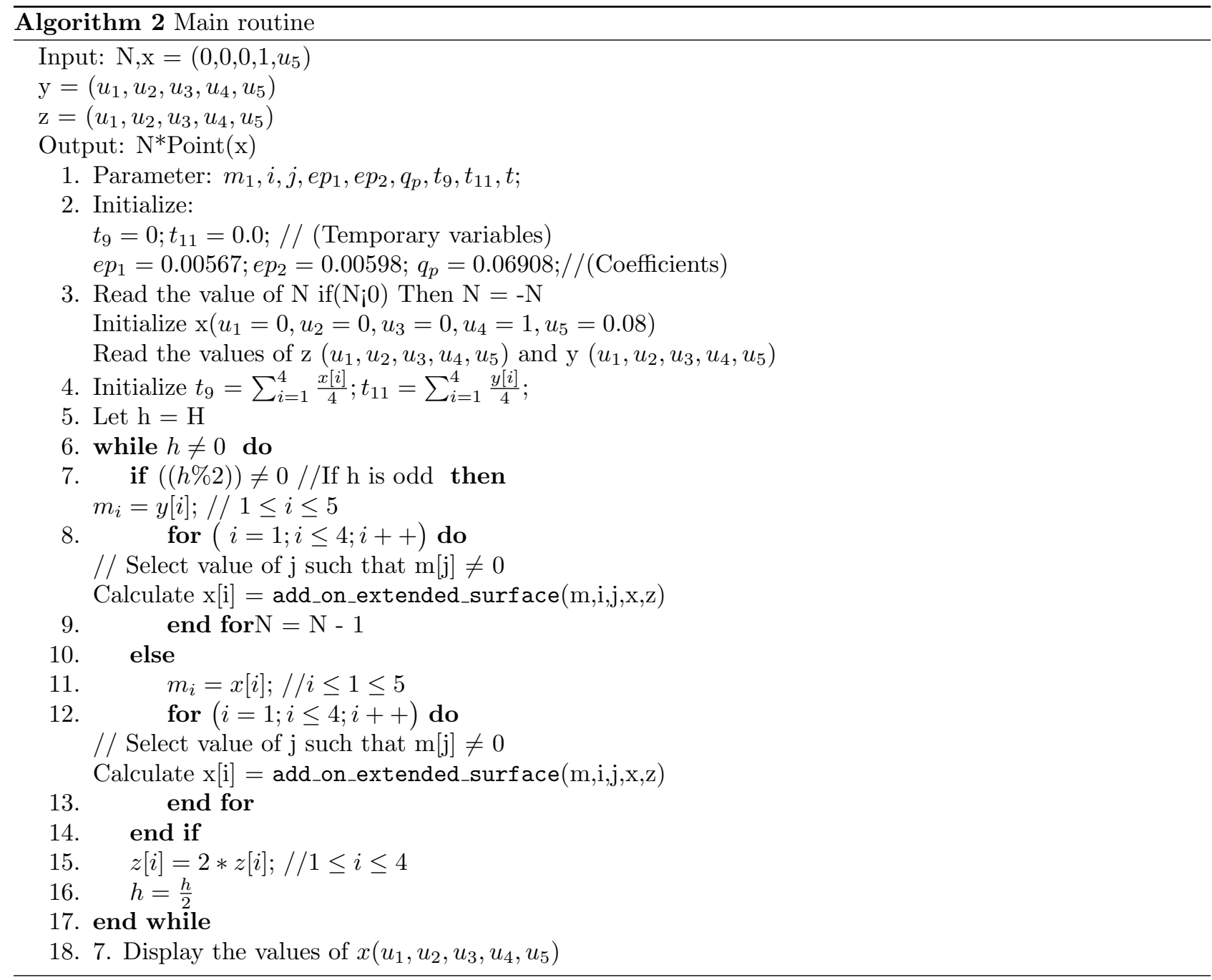

2.2. Using NSGA II for selecting a particular surface. The Kummer surface is described by the equation $[1]$ :

$$
y=\left(f_{6} \times x^{6}\right)+\left(f_{5} \times x^{5}\right)+\left(f_{4} \times x^{4}\right)+\left(f_{3} \times x^{3}\right)+\left(f_{2} \times x^{2}\right)+\left(f_{1} \times x^{1}\right)+f_{0}
$$

The next section describes different sample surfaces. An application of NSGA II for formulating multi-objective functions using different combinations of these surfaces is given next. NSGA II is an example of evolutionary algorithm designed for multi-objective optimization [28]. NSGA II could also be employed to make the decision about a particular surface.The different multi-objective equations with corresponding outputs are described below.

2.2.1. Multi-Objective Program 1. The multi-objective program 1 contains objectives $Z_{1}$ and $Z_{2}$ for two selected surfaces, which uses a parameter $e p_{1}$. The output obtained by running NSGA II for these objectives is shown in Fig. 2.1.

The initial values of parameters and equations used for objectives are given below:

- Coefficients $\left(f_{i}\right)$ used in surface 1 are given below:

$$
f_{0}=100, f_{1}=5, f_{2}=0.8, f_{3}=-1.87063, f_{4}=0.06, f_{5}=0.0, f_{6}=0.0, \text { and } e p_{1}=6.089
$$



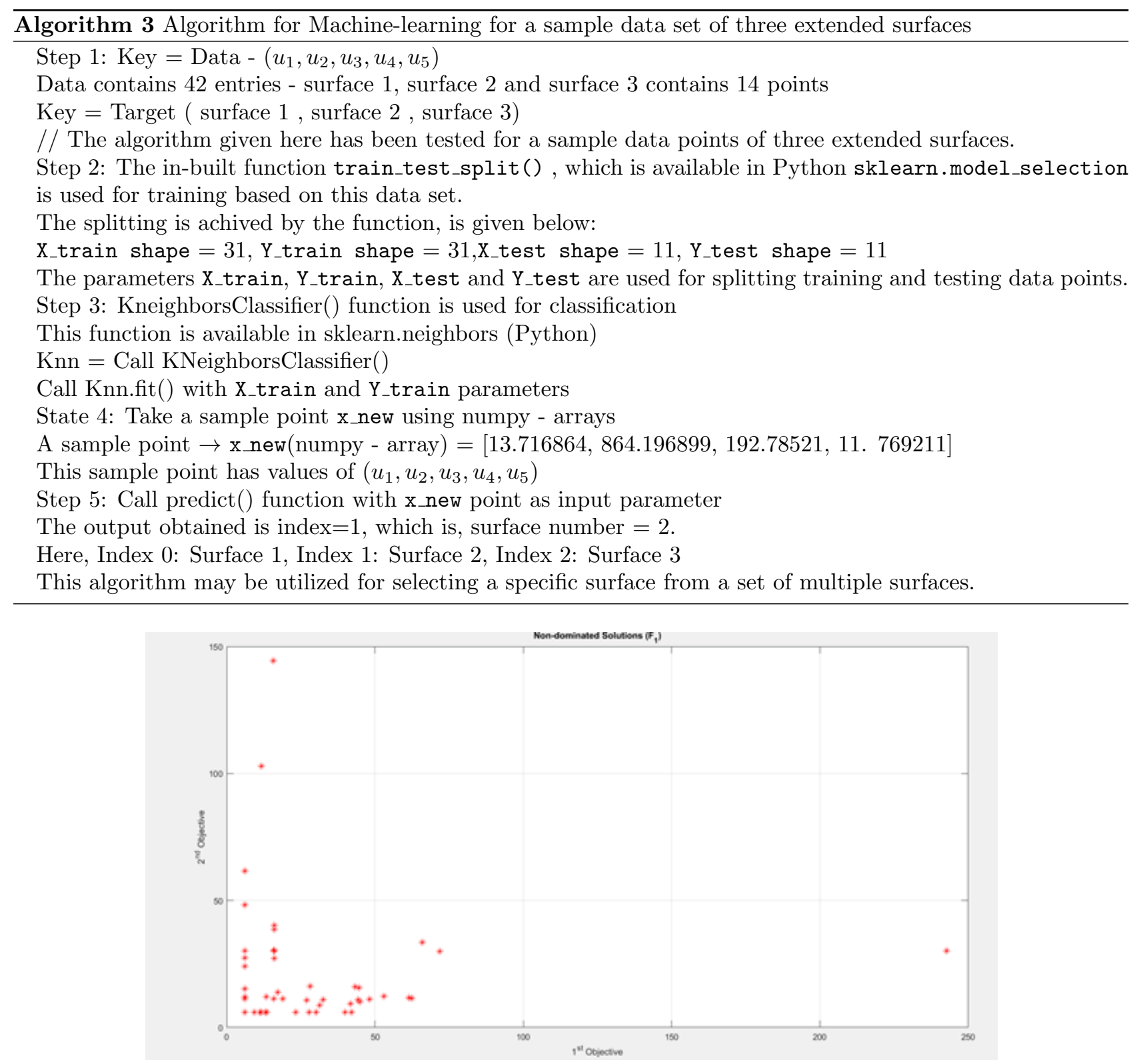

FIg. 2.1. Output of Multi-Objective Program 1

- Coefficients $\left(f_{i}\right)$ used in surface 2 are given below:

$$
f_{02}=120, f_{12}=3, f_{22}=0.65, f_{32}=-1.87063, f_{42}=0.08, f_{52}=0.09, f_{62}=0.00085
$$

- Objective 1:

$$
z_{1}=e p_{1}+\sqrt{\left(\left(f_{6} \times x^{6}\right)+\left(f_{5} \times x^{5}\right)+\left(f_{4} \times x^{4}\right)+\left(f_{3} \times x^{3}\right)+\left(f_{2} \times x^{2}\right)+\left(f_{1} \times x^{1}\right)+f_{0}\right)}
$$

- Objective 2:

$$
z_{2}=\sqrt{\left(\left(f_{62} \times x^{6}\right)+\left(f_{52} \times x^{5}\right)+\left(f_{42} \times x^{4}\right)+\left(f_{32} \times x^{3}\right)+\left(f_{22} \times x^{2}\right)+\left(f_{12} \times x^{1}\right)+f_{02}\right)}
$$


2.2.2. Multi-Objective Program 2. The multi-objective program 2 contains objectives $Z_{1}$ and $Z_{2}$ for two selected surfaces, which uses a parameter $e p_{1}$. The parameter of $f_{1}$ of surface 1 has been changed here, as compared to multi-objective program 1. The output obtained by running NSGA II for these objectives is shown in Fig.2.2.

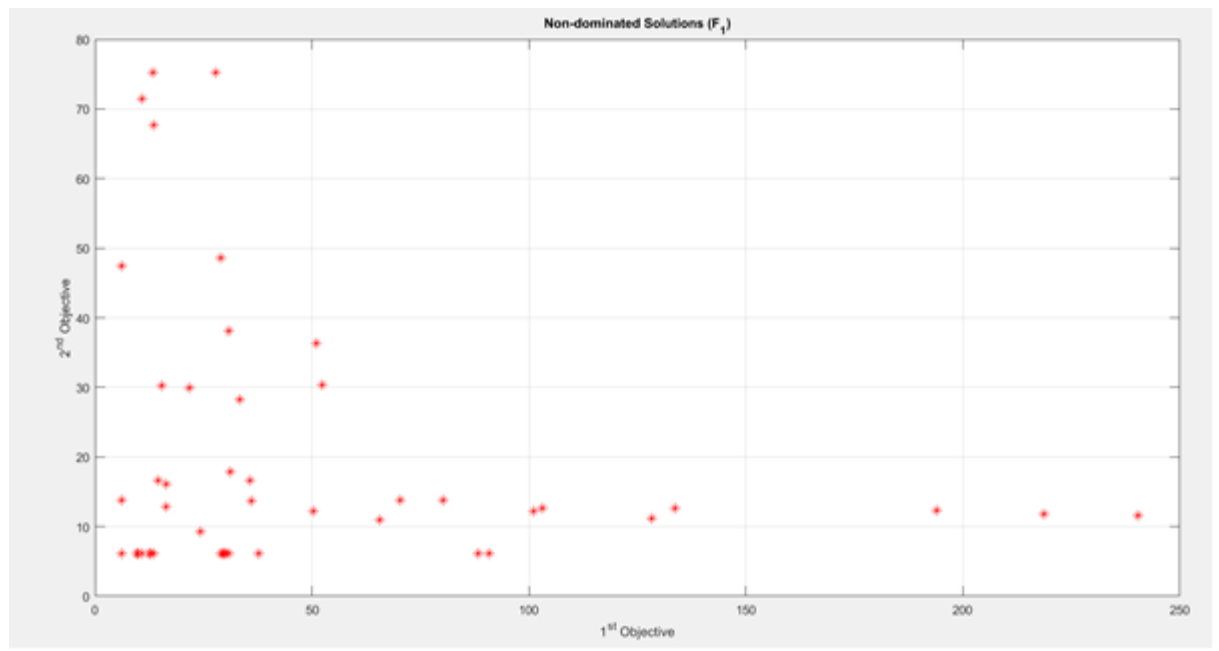

FIG. 2.2. Output of Multi-Objective Program 2

The initial values of parameters and equations used for objectives are given below:

- Coefficients $\left(f_{i}\right)$ used in surface 1 are given below:

$$
f_{0}=100, f_{1}=0.0, f_{2}=0.8, f_{3}=-1.87063, f_{4}=0.06, f_{5}=0.0, f_{6}=0.0, \text { and } e p_{1}=6.089
$$

- Coefficients $\left(f_{i}\right)$ used in surface 2 are given below:

$$
f_{02}=120, f_{12}=3, f_{22}=0.65, f_{32}=-1.87063, f_{42}=0.08, f_{52}=0.09, f_{62}=0.00085
$$

- Objective 1:

$$
z_{1}=e p_{1}+\sqrt{\left(\left(f_{6} \times x^{6}\right)+\left(f_{5} \times x^{5}\right)+\left(f_{4} \times x^{4}\right)+\left(f_{3} \times x^{3}\right)+\left(f_{2} \times x^{2}\right)+\left(f_{1} \times x^{1}\right)+f_{0}\right)}
$$

- Objective 2:

$$
z_{2}=\sqrt{\left(\left(f_{62} \times x^{6}\right)+\left(f_{52} \times x^{5}\right)+\left(f_{42} \times x^{4}\right)+\left(f_{32} \times x^{3}\right)+\left(f_{22} \times x^{2}\right)+\left(f_{12} \times x^{1}\right)+f_{02}\right)}
$$

2.2.3. Multi-Objective Program 3. The multi-objective program 3 contains objectives $Z_{1}$ and $Z_{2}$ for selected surface, which uses a parameter $e p_{1}$. Here, objective 1 uses two different parameters $q_{1}$ and $M_{i}$, it is described by equation (6). The output obtained by running NSGA II for these objectives is shown in Fig. 2.3.

The initial values of parameters and equations used for objectives are given below:

- $e p_{1}=6.089, M_{i}=120, q_{1}=7.41$

- Coefficients $\left(f_{i}\right)$ used in surface 2 are given below:

$$
f_{02}=120, f_{12}=3, f_{22}=0.65, f_{32}=-1.87063, f_{42}=0.08, f_{52}=0.09, f_{62}=0.00085
$$

- Objective 1:

$$
z_{1}=a b s\left(e p_{1}+q_{1} \times M_{i}^{2}+q_{1} \times M_{i} \times x^{2}\right)
$$

- Objective 2:

$$
z_{2}=\sqrt{\left(\left(f_{62} \times x^{6}\right)+\left(f_{52} \times x^{5}\right)+\left(f_{42} \times x^{4}\right)+\left(f_{32} \times x^{3}\right)+\left(f_{22} \times x^{2}\right)+\left(f_{12} \times x^{1}\right)+f_{02}\right)}
$$




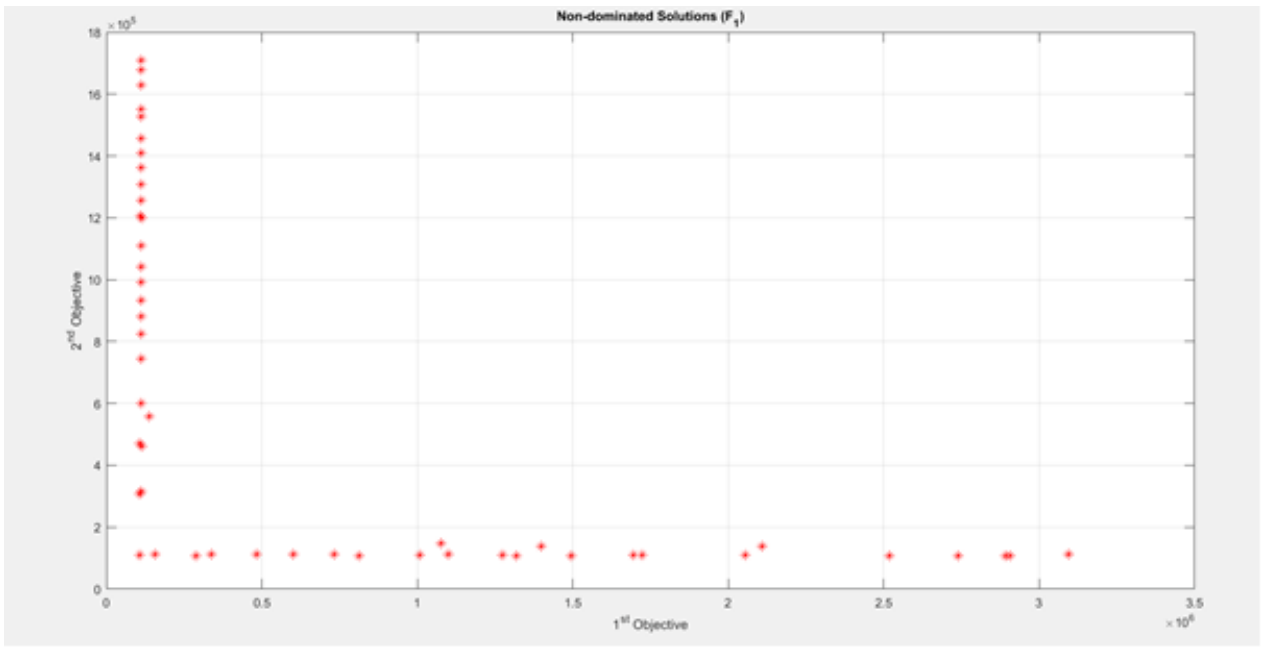

FIG. 2.3. Output of Multi-Objective Program 3

2.2.4. Multi-Objective Program 4. The multi-objective program 4 contains objectives $Z_{1}$ and $Z_{2}$ for selected surface, which uses a parameter $e p_{1}$. Here, objective 1 uses two different parameters $q_{1}$ and $M_{i}$, it is described by equation (8) and objective 2 has different parameters, as compared to multi-objective program 3. The output obtained by running NSGA II for these objectives is shown in Fig. 2.4.

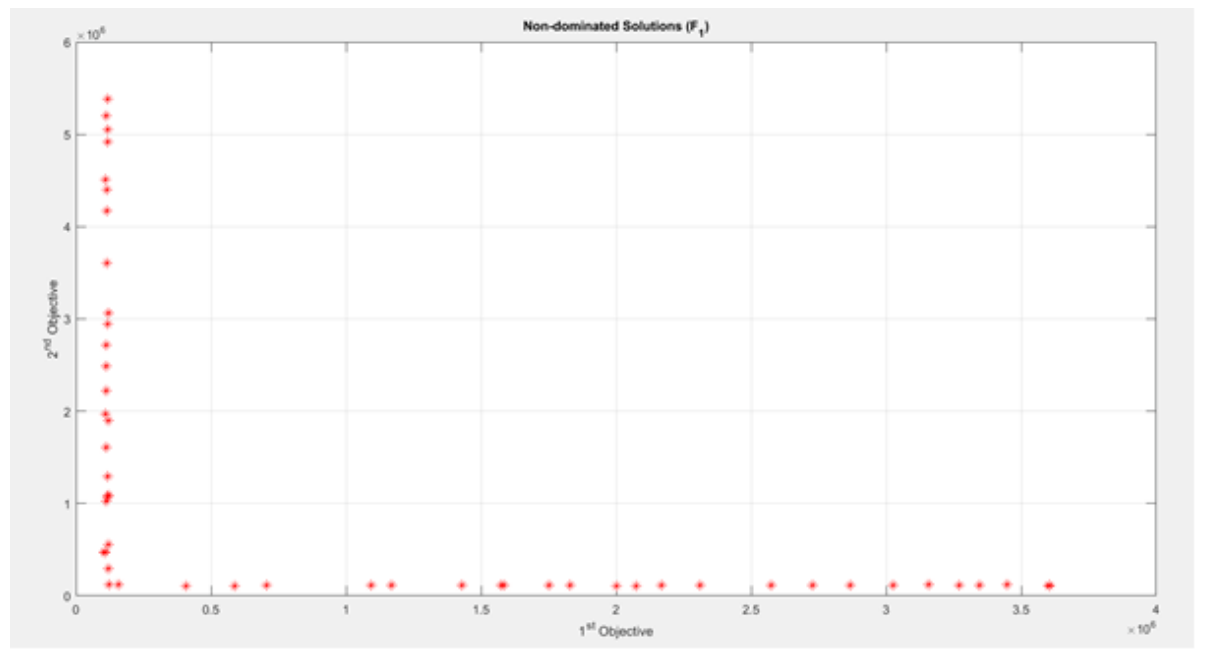

FIG. 2.4. Output of Multi-Objective Program 4

The initial values of parameters and equations used for objectives are given below:

- Coefficients $\left(f_{i}\right)$ used in surface 1 are $f_{0}=100, f_{1}=0.0, f_{2}=0.8, f_{3}=-1.87063, f_{4}=0.06, f_{5}=0.0, f_{6}=0.0$ and $e p_{1}=6.089, M_{i}=120, q_{1}=7.41$

- Objective 1:

$$
z_{1}=\operatorname{abs}\left(e p_{1}+q_{1} \times M_{i}^{2}+q_{1} \times M_{i} \times x^{2}\right)
$$

- Objective 2:

$$
z_{2}=\sqrt{\left(\left(f_{62} \times x^{6}\right)+\left(f_{52} \times x^{5}\right)+\left(f_{42} \times x^{4}\right)+\left(f_{32} \times x^{3}\right)+\left(f_{22} \times x^{2}\right)+\left(f_{12} \times x^{1}\right)+f_{02}\right)}
$$


TABLE 2.1

Sample input string composed of data streams of portfolio selection data that is used in SHA1 algorithm

\begin{tabular}{|l|l|l|l|l|l|}
\hline S.No. & \multicolumn{5}{|c|}{ Input String used in SHA1 algorithm } \\
\hline & Expected Return & Risk & Parameter $T_{a}$ & Parameter $B_{5}$ & Parameter $B_{10}$ \\
\hline 1. & "0.2560 & 0.1622 & 0.023 & 0.5628 & $0.4372 "+$ \\
\hline & "0.2786 & 0.1641 & 0.023 & 0.5002 & $0.4998 "+$ \\
\hline & "0.3012 & 0.1698 & 0.023 & 0.4377 & $0.5623 "+$ \\
\hline & "0.3238 & 0.1788 & 0.023 & 0.3752 & $0.6248 "+$ \\
\hline & "0.3464 & 0.1907 & 0.023 & 0.3126 & $0.6874 "+$ \\
\hline & "0.3690 & 0.2050 & 0.023 & 0.2501 & $0.7499 "+$ \\
\hline & "0.3916 & 0.2050 & 0.023 & 0.1876 & $0.8124 "+$ \\
\hline & "0.4142 & 0.2390 & 0.023 & 0.1251 & $0.8749 "+$ \\
\hline & "0.4368 & 0.2579 & 0.023 & 0.0438 & $0.9259 "+$ \\
\hline & "0.4594 & 0.2779 & 0.023 & 0.0 & $1.0 "$ \\
\hline
\end{tabular}

2.3. Using SHA-1 for authentication . For authentication SHA-1 algorithm could be utilized and a sample run with portfolio data is given in Table 2.1. Where, parameter $T_{a}$ and parameters $B_{5}, B_{10}$ are different values which are used in portfolio selection problem.

The output obtained after giving the input string, which is described in Table 2.1, to SHA1 algorithm is "DCF5C49BB3160A70788A72A06318FB2BE69BB233".

2.4. Cryptanalysis of the proposed protocol. It is proved that discrete logarithm solution for Kummer surface is equivalent to discrete logarithm solution for Jacobian [1]. With the additional point $u_{5}$ the security of the proposed protocol has not been lost moreover an additional direction for ensuring security has been included. The intension is to make the proposed protocol more secure with the help of new point $u_{5}$. The encryption scheme based on proposed protocol may utilize machine-learning methods for selecting a specified surface in the case of multiple surfaces. The machine learning algorithm has been tested on a sample data of three extended surfaces. As suggested in [1], ELGamal scheme for encryption may be designed that is based on calculation on the Kummer surface. The addition function provided here could be used for multiplication on the extended surface. Thus, the use of extended surface does not account for any security losses.

3. Generating keys of each round of AES using content-based matrix. This section provides details about the new scheme that is being used for key generation (AES). An overview of the structure of content-based matrix using sample data of portfolio selection is also given in Fig 3.1.

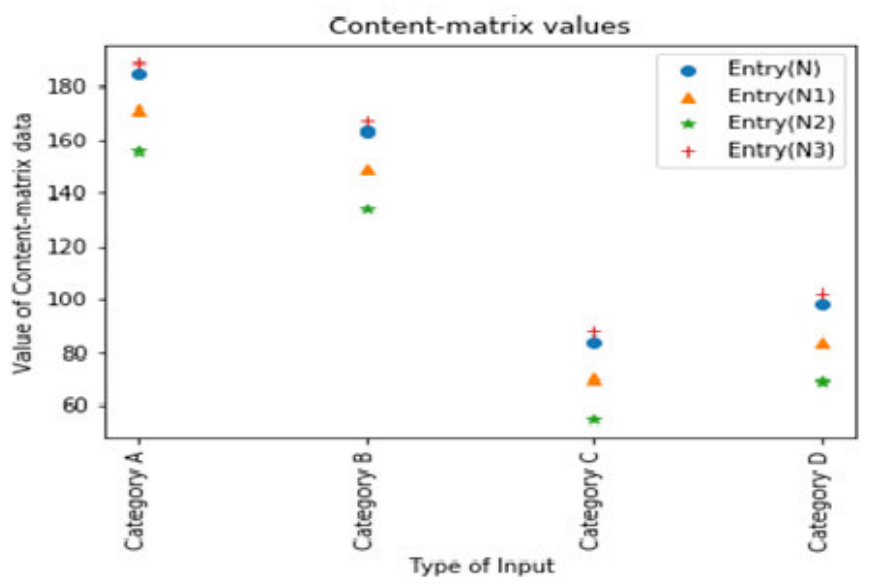

FIG. 3.1. Content-matrix values 
The formulation of the content-matrix is given next. Here, the input is divided into four categories- category A for characters a-z / A-Z, category B for numbers 0-9, category C for special characters and lastly, the category D for images / videos etc.

Here, a sample frequency distribution of different classes in a sample input is given in Table 3.1. It contains assumed values of weight $(\mathrm{x})$ and the mapping output $(\mathrm{y})$ is found using the following equation:

$$
\begin{aligned}
& y=x^{3}+x^{2}+w \\
& N_{o}=y \bmod 255
\end{aligned}
$$

The following constants are used here: $e p k_{1}=13.89, e p k_{2}=29.17$ and $e p k_{3}=-4.089$. The values of $N_{1}$, $N_{2}$ and $N_{3}$ are computed as described below:

$$
\begin{aligned}
& N_{1}=N-e p k_{1} \\
& N_{2}=N-e p k_{2} \\
& N_{3}=N-e p k_{3}
\end{aligned}
$$

\begin{tabular}{|c|c|c|c|c|c|c|c|c|}
\hline S.No. & Type of input & Frequency $(\mathrm{x})$ & Weight(w) & Mapping output(y) & Entry $\left(N_{0}\right)$ & $\operatorname{Entry}\left(N_{1}\right)$ & $\operatorname{Entry}\left(N_{2}\right)$ & $\operatorname{Entry}\left(N_{3}\right)$ \\
\hline 1 & Category A & 34 & 15 & 40475 & 185 & 171.11 & 155.825 & 189.089 \\
\hline 2 & Category B & 50 & 8 & 6252508 & 163 & 149.11 & 133.825 & 167.089 \\
\hline 3 & Category $\mathrm{C}$ & 60 & 9 & 216069 & 84 & 70.11 & 54.825 & 88.089 \\
\hline 4 & Category D & 12 & 11 & 1883 & 98 & 84.11 & 68.825 & 102.089 \\
\hline
\end{tabular}

TABLE 3.1

Output of content-matrix

The reduced content-matrix values are based upon only three categories: category-alpha $a_{1}=$ category 1 , category-alpha $a_{2}=$ category 2 and category-alpha $a_{3}=$ category 3 . The output obtained are given in Table 3.2 and Fig. 3.2.

TABLE 3.2

Output of content-matrix

\begin{tabular}{|c|c|c|c|c|c|}
\hline S.No. & Type of input & $\operatorname{Entry}(N)$ & $\operatorname{Entry}\left(N_{1}\right)$ & $\operatorname{Entry}\left(N_{2}\right)$ & $\operatorname{Entry}\left(N_{3}\right)$ \\
\hline 1 & Category- $\alpha_{1}$ & 185 & 171.11 & 155.825 & 189.089 \\
\hline 2 & Category- $\alpha_{2}$ & 163 & 149.11 & 133.825 & 167.089 \\
\hline 3 & Category- $\alpha_{3}$ & 91 & 77.11 & 61.825 & 95.089 \\
\hline
\end{tabular}

The following equations are used to compute optimal values :

$$
\begin{gathered}
\text { cost }_{1}=a_{1} \alpha_{1}^{2}+b_{1} \alpha_{1}+c_{1} \\
\text { cost }_{2}=a_{2} \alpha_{2}^{2}+b_{2} \alpha_{2}+c_{2} \\
\text { cost }_{3}=a_{3} \alpha_{3}^{2}+b_{3} \alpha_{3}+c_{3} \\
\text { Total_cost }=\text { cost }_{1}+\text { cost }_{2}+\text { cost }_{3} \\
189.089 \leq a_{1} \leq 155.825 \\
167.089 \leq a_{2} \leq 133.825 \\
95.089 \leq a_{3} \leq 61.825 \\
461.0 \leq \text { Total_cost } \leq 361.0
\end{gathered}
$$

The optimized values obtained after running classical Lagrangian multiplier method with sample inputvalues of $a_{i}, b_{i}$ and $c_{i}$ and using the inequality constraints values of $a_{1}, a_{2}$ and $a_{3}$ described above, are presented in Table 3.3 and Fig. 3.3. 


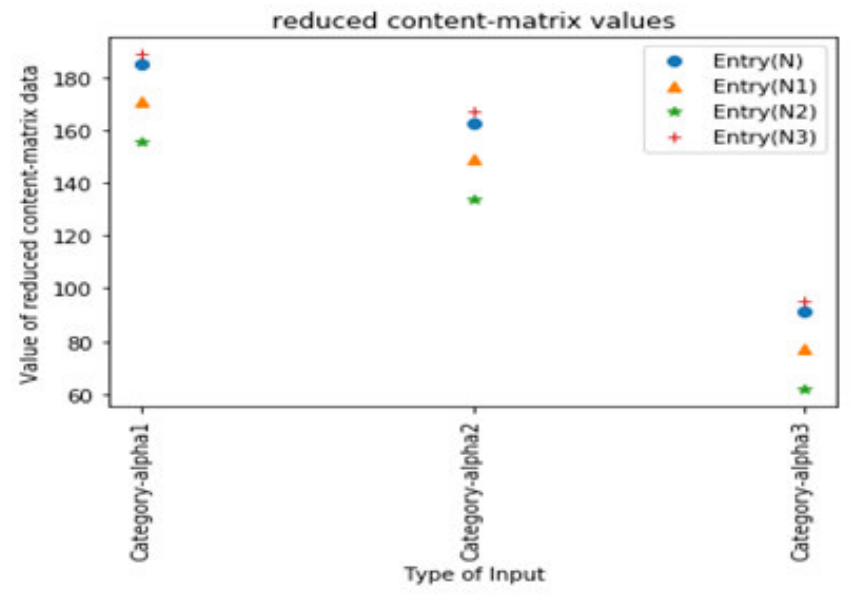

FIG. 3.2. Reduced content-matrix values

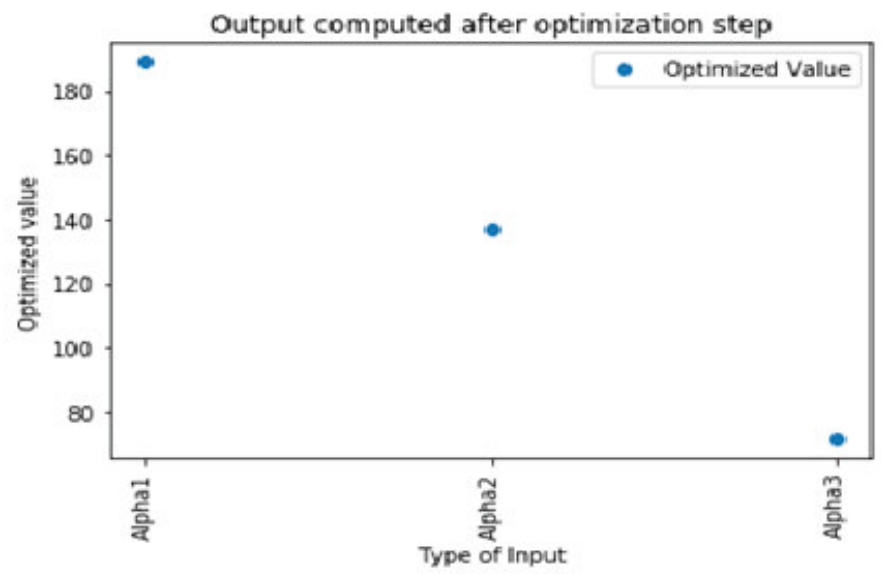

FIG. 3.3. Output computed after optimization step

TABLE 3.3

Optimized values of different categories

\begin{tabular}{|c|c|c|}
\hline \hline S.No. & Category & Optimized value \\
\hline \hline 1 & Category- $\alpha_{1}$ & 189.0809 \\
\hline 2 & Category- $\alpha_{2}$ & 137.2910 \\
\hline 3 & Category- $\alpha_{3}$ & 71.6765 \\
\hline
\end{tabular}

3.1. Cryptanalysis of the proposed scheme. Different kinds of cryptanalysis for AES (Rijndael) is provided in [28]. Using another technique based on calculating partial sum, the complexity of AES can be decreased [28]. The attacks on 7 and 8 rounds can be achieved by incorporating the information about extra texts. The next area to be considered is Key generation. Various undesired behaviour for key generation is discussed in [28].

This paper has made presentation about a novel scheme that may be adopted to conceal the information which could prove fruitful in increasing complexity of Key generation. Specifically, the structure of content-based matrix and selecting optimized values play a vital role in hiding the information. Since Key generation has a small number of non-linear components, the schemes presented here has given new dimension in this direction. 


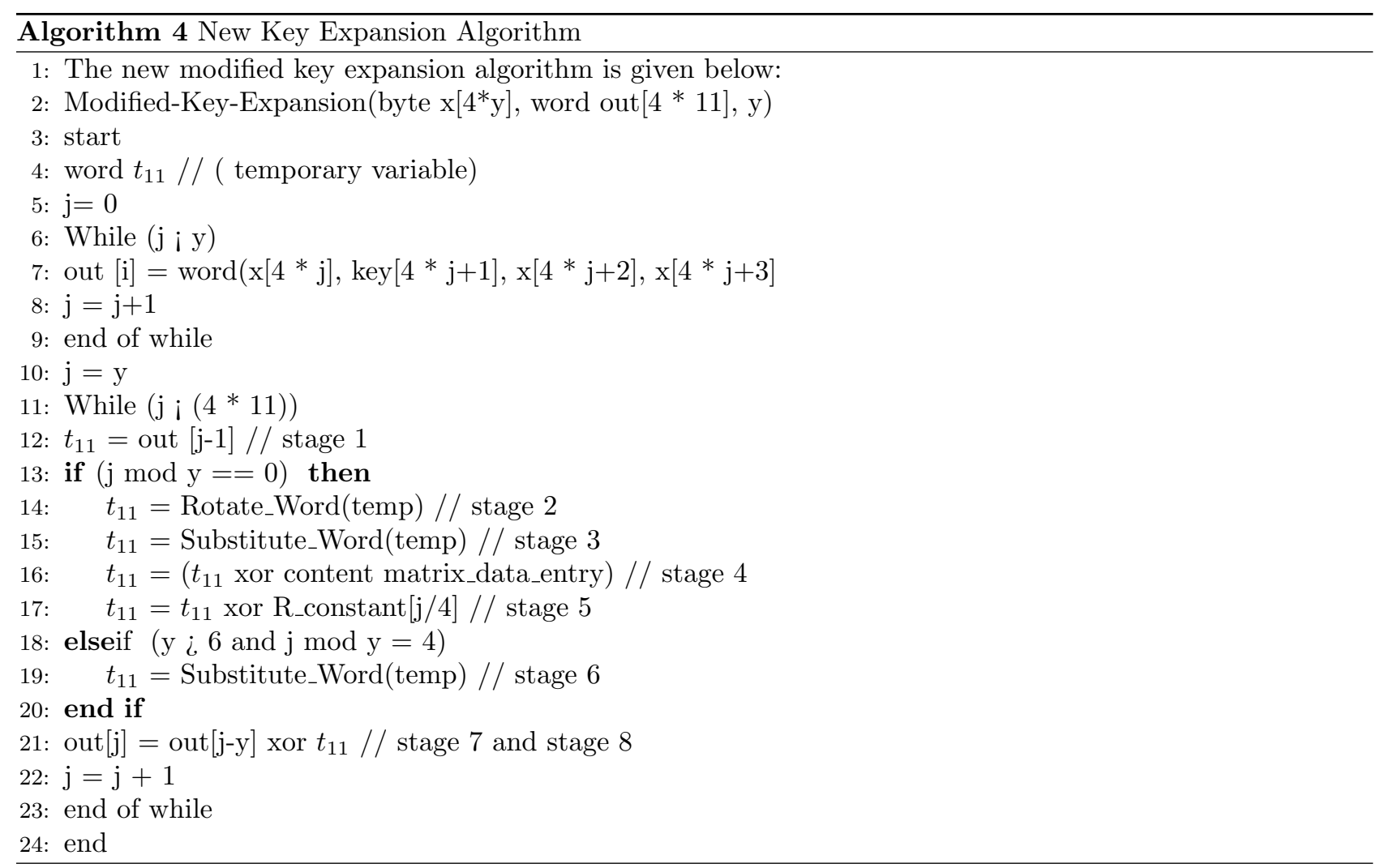

4. Result Analysis and Discussion. The following section describes the various outputs obtained after executing extended Kummer surface algorithm explained in section 2.1. An overview of the results obtained for existing Kummer surface is also given. The next section has a description of data obtained after executing new key expansion (AES) algorithm. Lastly, an overview of the results obtained with LIM algorithm are provided.

4.1. Existing Kummer Surface Results. This section briefly presents results which are obtained for existing and extended Kummer surface. The results are given in Table 4.1 and Fig 4.1 for existing Kummer surface- surface 1.

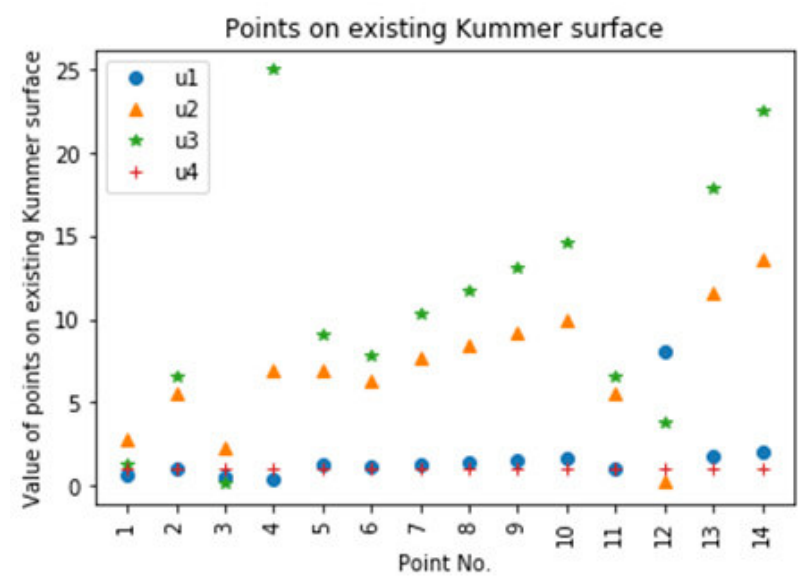

FIG. 4.1. Points on existing Kummer surface (Surface 1) 
TABLE 4.1

Sample points on Existing Kummer Surface $\left(u_{1}, u_{2}, u_{3}, u_{4}, u_{5}\right)($ Surface 1)

\begin{tabular}{|c|c|c|c|c|}
\hline \hline S.No. & $u_{1}$ & $u_{2}$ & $u_{3}$ & $u_{4}$ \\
\hline \hline 1 & 0.5915 & 2.7999 & 1.2000 & 1.0000 \\
\hline 2 & 1.0000 & 5.5856 & 6.5497 & 1.0000 \\
\hline 3 & 0.5229 & 2.2824 & 0.1000 & 1.0000 \\
\hline 4 & 0.4217 & 6.8683 & 25.0000 & 1.0000 \\
\hline 5 & 1.2000 & 6.9648 & 9.0642 & 1.0000 \\
\hline 6 & 1.1000 & 6.2700 & 7.7984 & 1.0000 \\
\hline 7 & 1.3000 & 7.6731 & 10.3607 & 1.0000 \\
\hline 8 & 1.4000 & 8.3982 & 11.7018 & 1.0000 \\
\hline 9 & 1.5000 & 9.1445 & 13.1036 & 1.0000 \\
\hline 10 & 1.6000 & 9.9176 & 14.5872 & 1.0000 \\
\hline 11 & 1.0000 & 5.5856 & 6.5460 & 1.0000 \\
\hline 12 & 8.0000 & 0.2864 & 3.7636 & 1.0000 \\
\hline 13 & 1.8000 & 11.5840 & 17.9428 & 1.0000 \\
\hline 14 & 2.0000 & 13.6073 & 22.5198 & 1.0000 \\
\hline
\end{tabular}

Similarly, sample data points are computed for surface 2 and surface 3.

4.1.1. Extended Kummer Surface Results. This section presents the results which are obtained after executing extended Kummer surface algorithm. This algorithm contains a newly added point $\left(u_{5}\right)$. The input points $\mathrm{z}$ and $\mathrm{y}$ on the extended Kummer surface (surface 1) are given in Table 4.2 and are drawn in Fig. 4.2 and Fig. 4.3.

TABLE 4.2

Value of input point (z) and input point (y) on extended Kummer surface (Surface 1)

\begin{tabular}{|c|c|c|c|c|c|c|c|c|c|c|}
\hline S.No. & $\mathrm{z}\left(u_{1}\right)$ & $\mathrm{z}\left(u_{2}\right)$ & $\mathrm{z}\left(u_{3}\right)$ & $\mathrm{z}\left(u_{4}\right)$ & $\mathrm{z}\left(u_{5}\right)$ & $\mathrm{y}\left(u_{1}\right)$ & $\mathrm{y}\left(u_{2}\right)$ & $\mathrm{y}\left(u_{3}\right)$ & $\mathrm{y}\left(u_{4}\right)$ & $\mathrm{y}\left(u_{5}\right)$ \\
\hline 1 & 1.0000 & 5.5856 & 6.5460 & 1.0000 & 0.8000 & 8.0000 & 0.2864 & 3.7636 & 1.0000 & 0.9800 \\
\hline 2 & 1.1000 & 6.2700 & 7.7984 & 1.0000 & 0.8000 & 1.3000 & 7.6731 & 10.3607 & 1.0000 & 0.9800 \\
\hline 3 & 1.2000 & 6.9648 & 9.0642 & 1.0000 & 0.8000 & 1.1000 & 6.2700 & 7.7984 & 1.0000 & 0.9800 \\
\hline 4 & 0.5915 & 2.7999 & 1.2000 & 1.0000 & 0.8000 & 1.0000 & 5.5856 & 6.5497 & 1.0000 & 0.9800 \\
\hline 5 & 8.0000 & 0.2864 & 3.7636 & 1.0000 & 0.8000 & 2.5033 & 13.0410 & 5.0000 & 1.0000 & 0.9800 \\
\hline 6 & 1.5000 & 9.1445 & 13.1036 & 1.0000 & 0.8000 & 1.6000 & 9.9176 & 14.5872 & 1.0000 & 0.9800 \\
\hline 7 & 0.4217 & 6.8683 & 25.0000 & 1.0000 & 0.8000 & 1.2000 & 6.9648 & 9.0642 & 1.0000 & 0.9800 \\
\hline 8 & 1.4000 & 8.3982 & 11.7018 & 1.0000 & 0.8000 & 1.5000 & 9.1445 & 13.1036 & 1.0000 & 0.9800 \\
\hline 9 & 0.5229 & 2.2824 & 0.1000 & 1.0000 & 0.8000 & 0.4217 & 6.8683 & 25.0000 & 1.0000 & 0.9800 \\
\hline 10 & 1.6000 & 9.9176 & 14.5872 & 1.0000 & 0.8000 & 1.0000 & 5.5856 & 6.5497 & 1.0000 & 0.9800 \\
\hline 11 & 1.0000 & 5.5856 & 6.5497 & 1.0000 & 0.8000 & 0.5229 & 2.2824 & 0.1000 & 1.0000 & 0.9800 \\
\hline 12 & 1.8000 & 11.5840 & 17.9428 & 1.0000 & 0.8000 & 2.0000 & 13.6073 & 22.5198 & 1.0000 & 0.9800 \\
\hline 13 & 1.3000 & 7.6731 & 10.3607 & 1.0000 & 0.8000 & 1.4000 & 8.3982 & 11.7018 & 1.0000 & 0.9800 \\
\hline 14 & 2.0000 & 13.6073 & 22.5198 & 1.0000 & 0.8000 & 0.5915 & 2.7999 & 1.2000 & 1.0000 & 0.9800 \\
\hline
\end{tabular}

Output points obtained are given in Table 4.3 and drawn in Fig.4.4. The data points obtained are sorted output according to point $\left(u_{5}\right)$. Similar outputs obtained for surface 2 and surface 3 . Moreover, the data points are sorted according to point $\left(u_{5}\right)$. An effort is made to present output points pictorially in ascending order of point $\left(u_{5}\right)$ such that the variation of point $\left(u_{5}\right)$ can be studied with values of other points $\left(u_{1}, u_{2}, u_{3}, u_{4}\right)$. A meaningful inference about the variation of point $\left(u_{5}\right)$ can be achieved with large set of input points. 


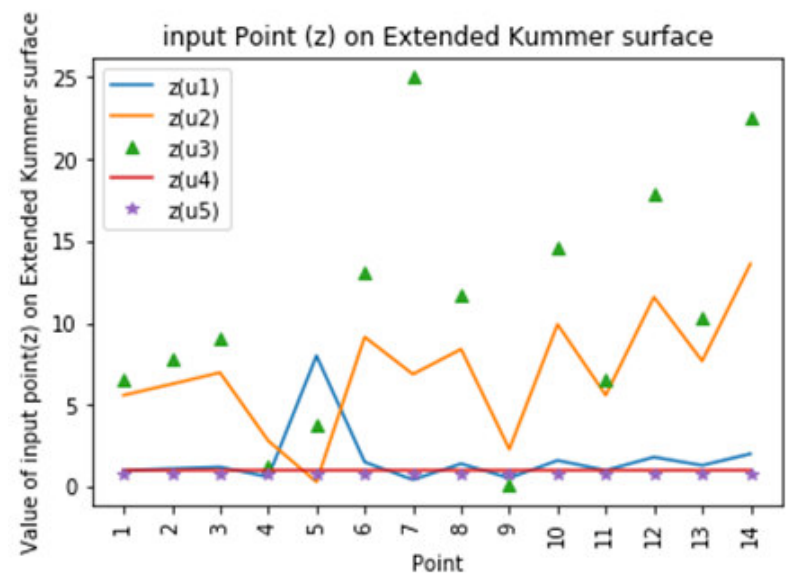

FIG. 4.2. Input point (z) on Extended Kummer surface (surface 1)

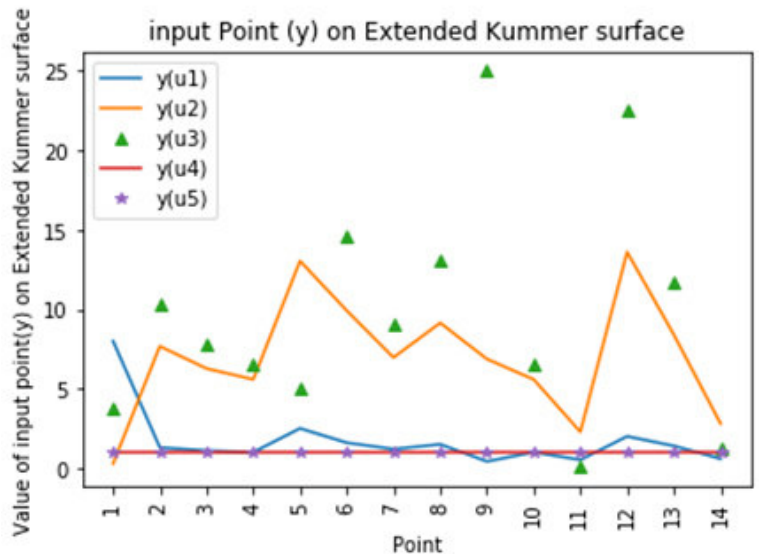

FIG. 4.3. Input point (y) on Extended Kummer surface (surface 1)

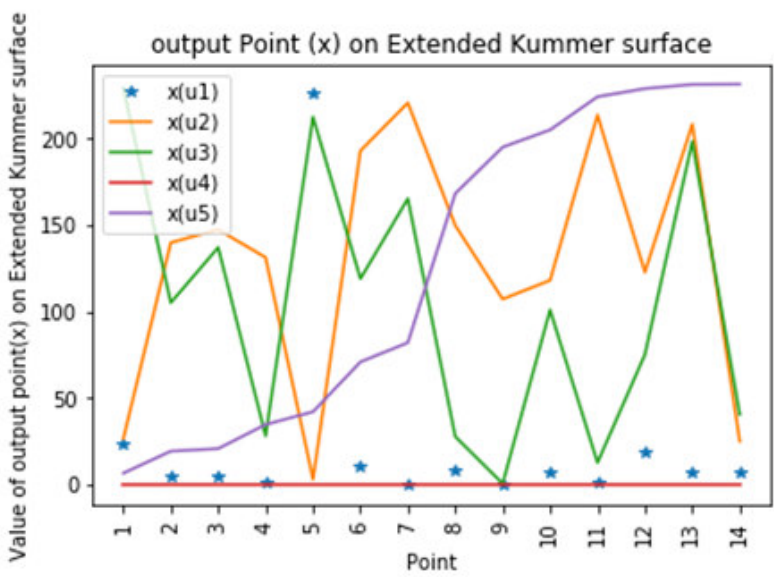

FIG. 4.4. Output point (x) Extended Kummer surface (surface 1) 
TABLE 4.3

Output values of extended Kummer Surface $\left(x\left(u_{1}\right), x\left(u_{2}\right), x\left(u_{3}\right), x\left(u_{4}\right), u_{5}\right)($ Surface 1)

\begin{tabular}{|c|c|c|c|c|c|}
\hline \hline S.No. & $x\left(u_{1}\right)$ & $x\left(u_{2}\right)$ & $x\left(u_{3}\right)$ & $x\left(u_{4}\right)$ & $x\left(u_{5}\right)$ \\
\hline \hline 1 & 24.0000 & 26.8055 & 229.3580 & 0.0000 & 6.7970 \\
\hline 2 & 4.7190 & 139.9495 & 105.2500 & 0.0000 & 19.4924 \\
\hline 3 & 4.7520 & 147.4385 & 137.1388 & 0.0000 & 20.9641 \\
\hline 4 & 1.0496 & 131.3605 & 28.2947 & 0.0000 & 34.8777 \\
\hline 5 & 225.6336 & 3.2089 & 212.4693 & 0.0000 & 42.2683 \\
\hline 6 & 10.8000 & 192.9697 & 119.0908 & 0.0000 & 71.0500 \\
\hline 7 & 0.6403 & 220.6695 & 165.3555 & 0.0000 & 82.1640 \\
\hline 8 & 8.8200 & 149.8854 & 27.9238 & 0.0000 & 168.2817 \\
\hline 9 & 0.3459 & 107.3347 & 0.7500 & 0.0000 & 195.0867 \\
\hline 10 & 7.6800 & 118.1619 & 101.0610 & 0.0000 & 205.0549 \\
\hline 11 & 1.5687 & 213.6205 & 12.8696 & 0.0000 & 224.2009 \\
\hline 12 & 19.4400 & 122.8515 & 75.2773 & 0.0000 & 228.8854 \\
\hline 13 & 7.0979 & 208.3531 & 198.3577 & 0.0000 & 231.2859 \\
\hline 14 & 7.0981 & 25.2546 & 40.7057 & 0.0000 & 231.4460 \\
\hline
\end{tabular}

4.2. Results obtained with new Key expansion algorithm in different stages. This section provides an overview of the outputs obtained after different stages in new Key expansion algorithm. For simplicity of the presented output, the decimal values on a scale of (value $/ 10^{9}$ ) is chosen. Table 4.4 has output of stage 4 and its (new Key expansion) diagram along with two trend lines - trade line 1(power trend line with forecast of 2.0 periods, equation used: $\mathrm{y}=34092 \times x^{00743}, R^{2}=0.1335$, here $\mathrm{y}$ is output, $\mathrm{x}$ is input parameter for trend line and $R^{2}$ is R-squared value used for trend line 1 ) and trend line 2 (polynomial trend with forecast of 2.0 periods) is given in Fig.4.5.

TABLE 4.4

Output data obtained from new Key expansion algorithm: Stage 4 and Stage 6

\begin{tabular}{|c|c|c|c|c|}
\hline S. No. & O(hex):Stage 4 & O(dec):Stage 4 & O(hex):Stgae 6 & O(dec):Stage 6 \\
\hline 1 & bf85ef55 & 3.2132 & be85ef55 & 3.1964 \\
\hline 2 & bdc5d7d4 & 3.1839 & bfc5d7d4 & 3.2174 \\
\hline 3 & fdd76f5f & 4.2588 & f9d76f5f & 4.1916 \\
\hline 4 & ff97ef7d & 4.2881 & f797ef7d & 4.1539 \\
\hline 5 & ffe5c7ff & 4.2932 & efe5c7ff & 4.0248 \\
\hline 6 & fdd5e6dc & 4.2587 & ddd5e6dc & 3.7218 \\
\hline 7 & bf954f5e & 3.2142 & ff954f5e & 4.2880 \\
\hline 8 & ffdfd75d & 4.2929 & 7fdfd75d & 2.1454 \\
\hline 9 & bff5ee5c & 3.2206 & a4f5ee5c & 2.7676 \\
\hline 10 & ffbdcf74 & 4.2906 & c9bdcf74 & 3.3847 \\
\hline
\end{tabular}

Table 4.4 has also output of stage 6 and its (new Key expansion) diagram along with two trend lines - trade line 1 (power trend line with forecast of 2.0 periods, equation used: $\mathrm{y}=3.7022 \times x^{0.049}, R^{2}=0.0266$ ) and trend line 2 (polynomial trend with forecast of 2.0 periods) is given in Fig.4.6.

In Table 4.4, O(hex):Stage 4 denotes Output of new Key expansion algorithm: stage 4 (Hex value), $\mathrm{O}(\mathrm{dec})$ :Stage 4 denotes Output of new Key expansion algorithm : stage 4 (Decimal value / 109 ), O(hex):Stage 6 denotes Output of new Key expansion algorithm : stage 6 (Hex value), and O(dec):Stage 6 denotes Output of new Key expansion algorithm : stage 6 (Decimal value $/ 10^{9}$ ).

In Table $4.5, \mathrm{O}$ (hex):Stage 7 denotes Output of new Key expansion algorithm : stage 7 (Hex value), $\mathrm{O}(\mathrm{dec})$ :Stage 7 denotes Output of new Key expansion algorithm : stage 7 (Decimal value / 10 ${ }^{9}$ ), O(hex):stage 


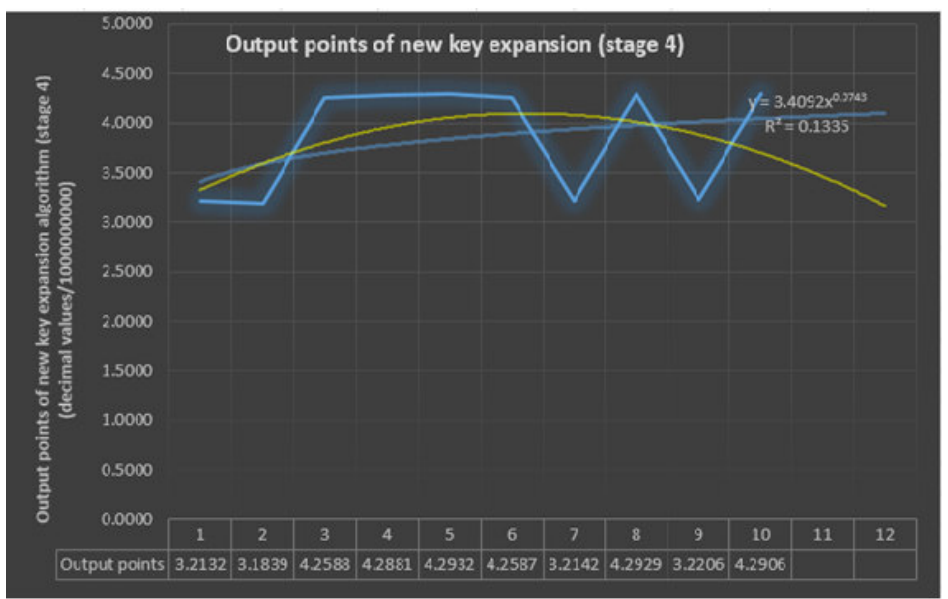

FIG. 4.5. Output points of new key expansion (stage 4)

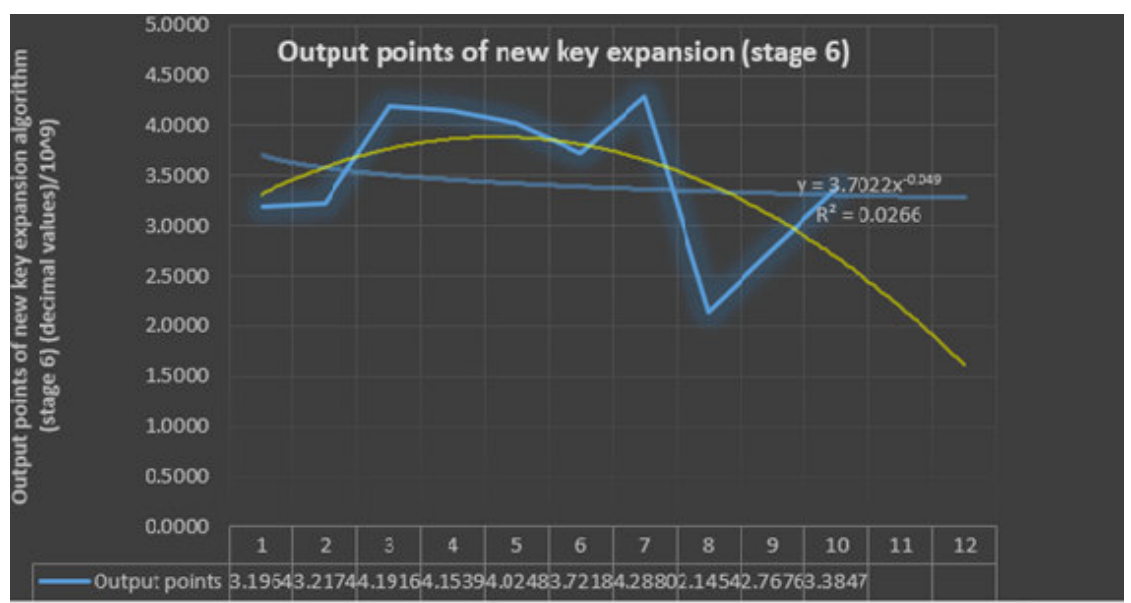

FIG. 4.6. Output points of new key expansion (stage 6)

8 denotes Output of new Key expansion algorithm : stage 8 (Hex value), and $\mathrm{O}(\mathrm{dec})$ :stage 8 denotes Output of new Key expansion algorithm : stage 8 (Decimal value / $10^{9}$ ).

Table 4.5 also has output of stage 7 and its (new Key expansion) diagram along with two trend lines trade line 1 (power trend line with forecast of 2.0 periods, equation used: $\mathrm{y}=0.9656 \times x^{0.0177}, R^{2}=0.0002$ ) and trend line 2 (polynomial trend with forecast of 2.0 periods) is given in Fig.4.7.

Table 4.5 has output of stage 8 and its (new Key expansion) diagram along with two trend lines-trade line 1 (power trend line with forecast of 2.0 periods, equation used: $y=0.9656 \times x^{0.0177}, R^{2}=0.0002$ ) and trend line 2 (polynomial trend with forecast of 2.0 periods) is given in Fig.4.8.

As evident from the outputs presented in Table 4.4 and Table 4.5, the trade lines are having different patterns in the existing and new Key expansion algorithm. The output of stage 1,2, 3 and 5 have same outputs in both existing and new Key expansion algorithm, and are given in Table 4.6. The stage 4 is available only in the new Key expansion. The outputs in the stage 6 are altogether different in new algorithm. The variation of points is different in new stage 7, whereas the trend lines are having similar patterns in this stage. Lastly, the stage 8 has different variation of points in new Key expansion, moreover trade lines depicts different behaviour. Thus, the proposed scheme adopted can be utilized by a designer in order to provide better security by having newer variation patterns, which are different from existing AES Key expansion algorithm. 
TABLE 4.5

Output data obtained from new Key expansion algorithm: Stage 7 and Stage 8

\begin{tabular}{|c|c|c|c|c|}
\hline S.No. & O(hex):Stage 7 & $\mathrm{O}(\mathrm{dec}):$ Stage 7 & O(hex):Stage 8 & $\mathrm{O}($ dec $):$ Stage 8 \\
\hline 1 & 2b7e1576 & 0.7297 & 2b7e1576 & 0.7297 \\
\hline 2 & 28 aed2a6 & 0.6825 & 28aed2a6 & 0.6825 \\
\hline 3 & abf71588 & 2.8851 & abf71588 & 2.8851 \\
\hline 4 & 09cf4f3c & 0.1646 & 09cf4f3c & 0.1646 \\
\hline 5 & $95 \mathrm{fbfa} 43$ & 2.5163 & $95 \mathrm{fbfa} 43$ & 2.5163 \\
\hline 6 & bd5528e 5 & 3.1765 & $\mathrm{bd} 5528 \mathrm{e} 5$ & 3.1765 \\
\hline 7 & $16 \mathrm{a} 23 \mathrm{~d} 6 \mathrm{~d}$ & 0.3797 & $16 \mathrm{a} 23 \mathrm{~d} 6 \mathrm{~d}$ & 0.3797 \\
\hline 8 & $1 \mathrm{f} 6 \mathrm{~d} 7251$ & 0.5273 & $1 \mathrm{f} 6 \mathrm{~d} 7251$ & 0.5273 \\
\hline 9 & $2 \mathrm{a} 3 \mathrm{e} 2 \mathrm{~d} 97$ & 0.7087 & 2a3e2d97 & 0.7087 \\
\hline 10 & $976 \mathrm{~b} 572$ & 0.1588 & $976 \mathrm{~b} 572$ & 0.1588 \\
\hline 11 & 81c9381f & 2.1774 & 81c9381f & 2.1774 \\
\hline 12 & $9 \mathrm{ea} 44 \mathrm{a} 4 \mathrm{e}$ & 2.6616 & 9ea44a4e & 2.6616 \\
\hline 13 & d3e942c8 & 3.5553 & d3e942c8 & 3.5553 \\
\hline 14 & $448247 \mathrm{ba}$ & 1.1494 & $448247 \mathrm{ba}$ & 1.1494 \\
\hline 15 & c54b7fa5 & 3.3101 & c54b7fa5 & 3.3101 \\
\hline 16 & 5bef35eb & 1.5424 & 5bef35eb & 1.5424 \\
\hline 17 & 247eadb5 & 0.6123 & 247eadb5 & 0.6123 \\
\hline 18 & 60fceaf & 0.1017 & 60fceaf & 0.1017 \\
\hline 19 & a5b795aa & 2.7803 & a5b795aa & 2.7803 \\
\hline 20 & fe58a041 & 4.2672 & fe58a041 & 4.2672 \\
\hline 21 & cb9b6a4a & 3.4160 & cb9b6a4a & 3.4160 \\
\hline 22 & ab678045 & 2.8757 & ab678045 & 2.8757 \\
\hline 23 & ed015ef & 0.2485 & ed015ef & 0.2485 \\
\hline 24 & f088b5ae & 4.0355 & f088b5ae & 4.0355 \\
\hline 25 & $164 \mathrm{e} 8 \mathrm{c} 96$ & 0.3742 & $164 \mathrm{e} 8 \mathrm{c} 96$ & 0.3742 \\
\hline 26 & bd29cd3 & 0.1984 & bd29cd3 & 0.1984 \\
\hline 27 & b3f9193c & 3.0194 & b3f9193c & 3.0194 \\
\hline 28 & $4371 \mathrm{ac} 92$ & 1.1315 & $4371 \mathrm{ac} 92$ & 1.1315 \\
\hline 29 & e9dbc3c8 & 3.9235 & e9dbc3c8 & 3.9235 \\
\hline 30 & 54f2cf1b & 1.4252 & $54 f 2 c f 1 b$ & 1.4252 \\
\hline 31 & e7bd627 & 0.2430 & e7bd627 & 0.2430 \\
\hline 32 & a47a7ab5 & 2.7595 & a47a7ab5 & 2.7595 \\
\hline 33 & 9641495 & 0.1576 & 9641495 & 0.1576 \\
\hline 34 & $\mathrm{c} 2 \mathrm{f} 6 \mathrm{db} 8 \mathrm{e}$ & 3.2710 & c2f6db8e & 3.2710 \\
\hline 35 & 25fdda9 & 0.0398 & 25fdda9 & 0.0398 \\
\hline 36 & 8187771c & 2.1731 & $8187771 c$ & 2.1731 \\
\hline 37 & 32f1fac9 & 0.8547 & 32f1fac9 & 0.8547 \\
\hline 38 & f072147 & 0.2521 & f072147 & 0.2521 \\
\hline 39 & d5fa2cee & 3.5899 & d5fa2cee & 3.5899 \\
\hline 40 & 547d5bf2 & 1.4175 & $547 \mathrm{~d} 5 \mathrm{bf} 2$ & 1.4175 \\
\hline
\end{tabular}

4.3. Output of LIM. This section presents computed output values of LIM obtained from histogram data taken from different extended Kummer surface. The values of LIM for data used in extended Kummer surface 1 is given in Table 4.7, the diagrams showing histograms for first five values (set 1) and last five values (set 2) of Table 4.7 are given in Fig.4.9 and Fig.4.10. The computed values of LIM for surface 1 are depicted in Fig. 4.11. The algorithm of LIM is presented in [10]. 


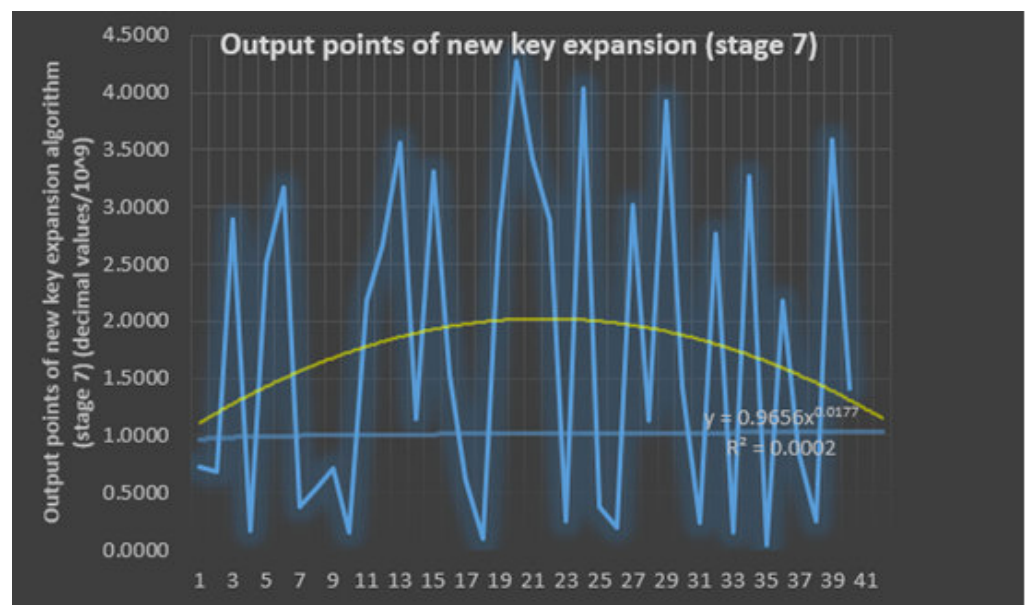

FIG. 4.7. Output points of new key expansion (stage 7)

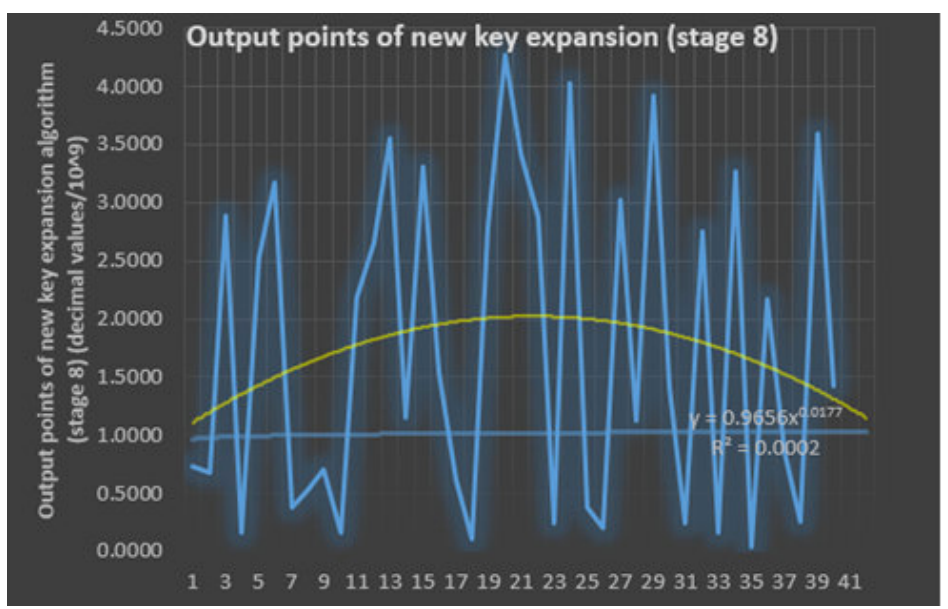

FIG. 4.8. Output points of new key expansion (stage 8)

TABLE 4.6

Output of stage 1, stage 2 and stage 3 (existing and new Key expansion)

\begin{tabular}{|c|c|c|c|}
\hline \hline S.No. & Variable t11 (stage 1) & Output after Rotate_word ( ) (stage 2) & Output after Subtitute_word ( ) (stage 3) \\
\hline \hline 1 & $09 \mathrm{cf4f3c}$ & cf4f3c09 & 8a84eb01 \\
\hline 2 & $1 \mathrm{f} 6 \mathrm{~d} 7251$ & $6 \mathrm{~d} 72511 \mathrm{f}$ & $3 \mathrm{c} 40 \mathrm{~d} 1 \mathrm{c} 0$ \\
\hline 3 & $9 \mathrm{ea} 44 \mathrm{a} 4 \mathrm{e}$ & $\mathrm{a} 44 \mathrm{a} 4 \mathrm{e} 9 \mathrm{e}$ & $49 \mathrm{~d} 62 \mathrm{fb}$ \\
\hline 4 & $5 \mathrm{bef35eb}$ & ef35eb5b & df96e939 \\
\hline 5 & fe58a041 & $58 \mathrm{a} 041 \mathrm{fe}$ & cae083bb \\
\hline 6 & f088b5ae & $88 \mathrm{~b} 5 \mathrm{aef} 0$ & $\mathrm{~d} 5914 \mathrm{c} \mathrm{c}$ \\
\hline 7 & $4371 \mathrm{ac} 92$ & $71 \mathrm{ac} 9243$ & dadad549 \\
\hline 8 & $\mathrm{a} 47 \mathrm{a} 7 \mathrm{ab} 5$ & $7 \mathrm{a} 7 \mathrm{ab} 5 \mathrm{a} 4$ & $17 \mathrm{f5acc}$ \\
\hline 9 & $8187771 \mathrm{c} 81$ & $87771 \mathrm{c} 81$ & ff398920 \\
\hline 10 & $547 \mathrm{~d} 5 \mathrm{bf} 2$ & $7 \mathrm{~d} 5 \mathrm{bf} 254$ & \\
\hline
\end{tabular}

As given in Table 4.7, Step 1 denotes Histogram Data (step 1), Step 3 denotes Sorted Data computed in the Algorithm (step 3), and Cval(LIM) denotes Computed value of LIM. The values of LIM for data used in extended Kummer surface 2 are 0.3451010 and 0.477174 for two different sample data points. Same values of LIM is obtained for surface 3 . 


\section{Histogram data used in LIM algorithm}

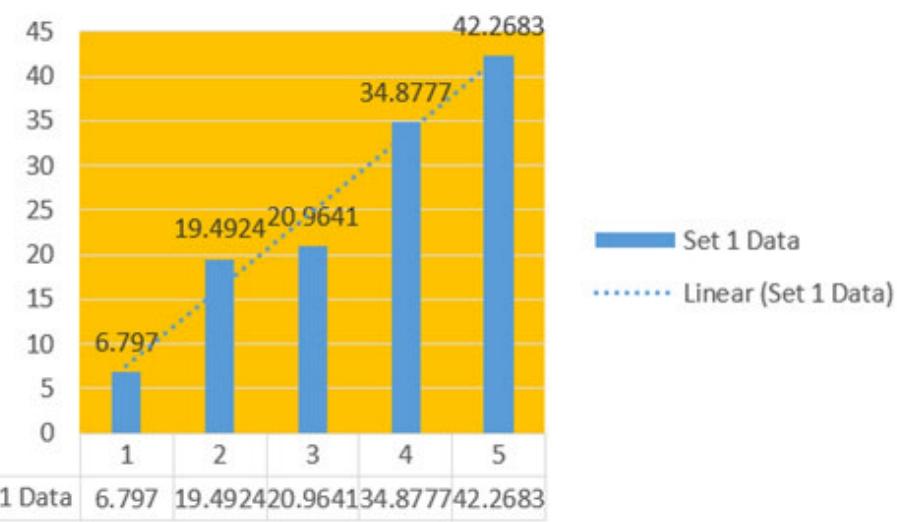

FIG. 4.9. Histogram data used in LIM algorithm (surface 1) (set 1 data)

Histogram data used in LIM algorithm

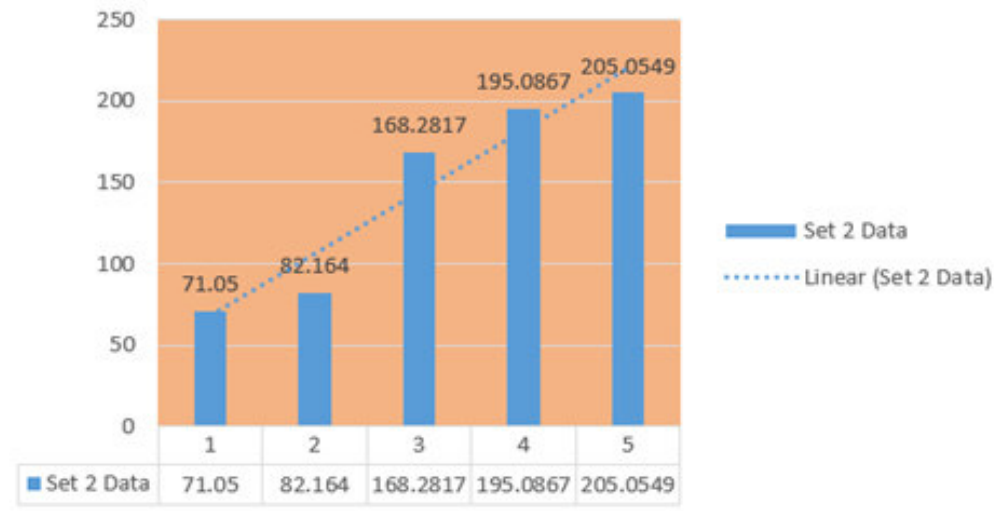

FIG. 4.10. Histogram data used in LIM algorithm (surface 1) (set 2 data)

\section{Computed values of LIM}

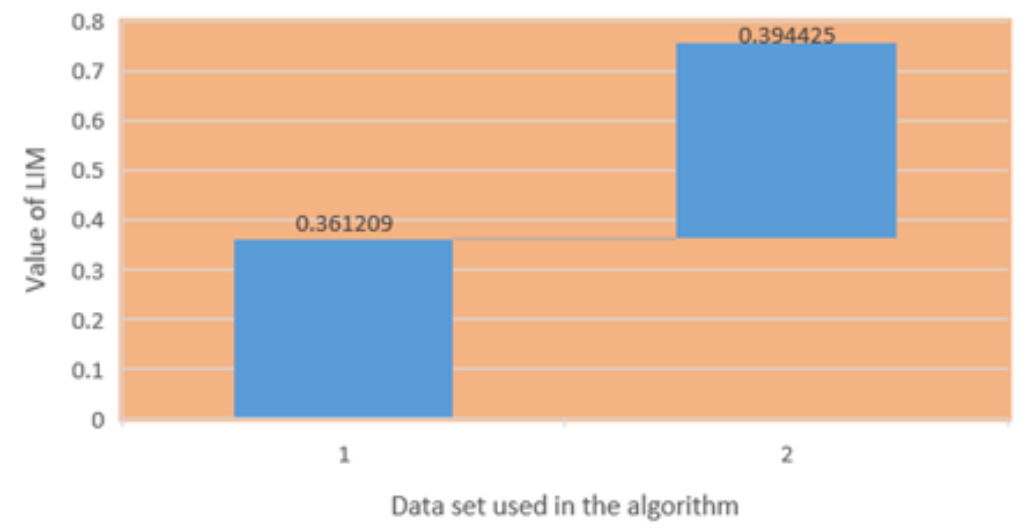

FIG. 4.11. Computed values of LIM (surface 1) 
TABLE 4.7

Output of LIM (surface 1)

\begin{tabular}{|c|c|c|c|}
\hline S. No. & Step 1 & Step 3 & Cval(LIM) \\
\hline 1 & 6.797 & 16.080608 & 0.361209 \\
\hline & 19.4924 & 46.115883 & \\
\hline & 20.9641 & 49.597691 & \\
\hline & 34.8777 & 82.51503 & \\
\hline & 42.2683 & 100 & \\
\hline 2 & 71.05 & 34.649258 & 0.394425 \\
\hline & 82.164 & 40.069271 & \\
\hline & 168.2817 & 82.06665 & \\
\hline & 195.0867 & 95.138763 & \\
\hline & 205.0549 & 100 & \\
\hline
\end{tabular}

Comparison of average values of $\mathrm{N}$ Blocks (LIM) for different Surfaces

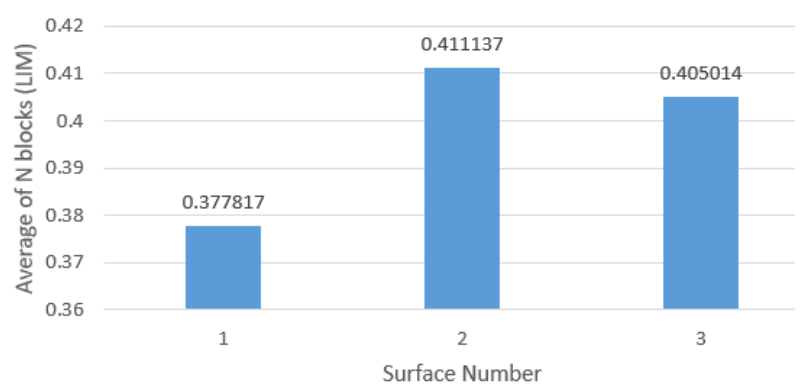

FIG. 4.12. Comparison of average values of $N$ Blocks (LIM) for different surfaces)

4.3.1. Importance of computed values of LIM. An encrypting method, which has randomly distributed cipher texts inside the group of text used while encrypting, would be classified as a potential nice method encrypting. A computed value of LIM, that is, close to a value of 0.5 is an indication of randomly distributed [10]. Moreover, a drift from this value, significance occurrence of a kind of pattern in the cipher text [10]. The average values shown in Fig. 4.12 signifies that all the three surfaces are different, and there is a possibility of occurrence of a pattern, because these values drift from the value of 0.5 .

5. Conclusion. This paper has a secure structure which contains mainly two aspects of a cryptographic system. Firstly, a scheme comprising of newly added point $\left(u_{5}\right)$ in the kummer surface and then how this information inclusion could be utilized for a machine-learning algorithm in selecting an appropriate surface. Here, the selection of a particular surface based on a machine learning technique is provided. The work based on kummer surfaces and building a Fast Diffie-Hellman protocol is previously given in earlier papers. The focus of the proposed scheme is to enhance the security of the above-mentioned work by selecting an additional point $\left(u_{5}\right)$ on the surface. Later, selection of multiple surfaces using NSGA II algorithm is given. Secondly, an encryption based on AES is taken. A new scheme for generating modified key-expansion in AES algorithm is described. The scheme builds a content-matrix using frequencies of different categories in the input message. Next, optimized values of the entries are chosen by running classical Lagrangian multiplier method. The selected entries are utilized in the modified key-expansion algorithm. Lastly, a brief overview of LIM index is given.

Acknowledgement: This publication is an outcome of the $\mathrm{R} \& \mathrm{D}$ work under taken project under the Visvesarvaraya PhD Scheme of Ministry of Electronics and IT, Government of India, being implemented by the Digital India Corporation. 


\section{REFERENCES}

[1] N. P. Smart, And S. Siksek, A Fast Diffie Hellman Protocol in Genus 2, in Journal of cryptology, vol.12(1), 1999, pp. 67-73.

[2] H. Nover, Algebraic cryptanalysis of AES: an overview, in University of Wisconsin, USA, (2005).

[3] L. Ning, L.Kanfeng, L. Wenliang, and D. Zhongliang, a joint encryption and error correction method used in satellite communications, in China communications, vol. 11(3), (2014),pp. 70-79.

[4] Y. Dodis, R. LEOnid, AND S. AdAM, Fuzzy extractors: How to generate strong keys from biometrics and other noisy data, in International conference on the theory and applications of cryptographic techniques, (2004), pp. 523-540.

[5] C. Peikert, and W. Brent, Lossy trapdoor functions and their applications, in SIAM Journal on Computing, vol.40(6), (2011), pp.1803-1844.

[6] A. C. YAO, Theory and application of trapdoor functions, in 23rd Annual Symposium on Foundations of Computer Science SFCS'08, (1982), pp.80-91.

[7] L. Blum, B. MAnuel, And S. Mike, A simple unpredictable pseudo-random number generator, in SIAM Journal on computing, vol. 15(2), (1986), pp.364-383.

[8] P. S. Mehra, M. N. Doja, and B. Alam, Codeword Authenticated Key Exchange (CAKE) light weight secure routing protocol for WSN, International Journal of Communication Systems, 32 (2019).

[9] R. Cramer, And S. Victor, A practical public key cryptosystem provably secure against adaptive chosen ciphertext attack, in Annual International Cryptology Conference, (1982), pp. 13-25.

[10] V. Karuvandan, C. Senthamarai and P. Shantharajah, in Cryptanalysis of AES-128 and AES-256 block ciphers using lorenz information measure, in Int. Arab J. Inf. Technology, vol. 13(6B), (2016), pp. 1054-1060.

[11] N. Ferguson, K. John, L. Stefan, S. Bruce, S. Mike, W.David, and W.Doug, Improved cryptanalysis of Rijndael, in International Workshop on Fast Software Encryption, 2000, pp. 213-230.

[12] A. Kaminsky, K. Michael, and R. Stanisaw, An overview of cryptanalysis research for the advanced encryption standard, in MILITARY COMMUNICATIONS CONFERENCE, 2010-MILCOM, (2010), pp.1310-1316.

[13] S. Chen, L. Rodolphe, L. Jooyoung, S. Yannick, and S. John, Minimizing the two-round EvenMansour cipher, in Journal of Cryptology, vol. 31(4), (2018), pp. 1064-1119.

[14] M. Masoumi, And M. HADI Rezayati, Novel approach to protect advanced encryption standard algorithm implementation against differential electromagnetic and power analysis, in IEEE Transactions on Information Forensics and Security, Vol. 10(2), 2015, pp.256-265.

[15] F. Courbon, JJ. A. Fournier, P. L. Moundi, And A. Tria, Combining image processing and laser fault injections for characterizing a hardware AES, in IEEE transactions on computer-aided design of integrated circuits and systems, vol. 34(6), (2015), pp.928-936.

[16] M. TAha, And P. SChaumont, Key updating for leakage resiliency with application to AES modes of operation, in IEEE transactions on information forensics and security, vol. 10(3), (2015), pp.519-528.

[17] C. H. BAEK, J.H.Cheon, AND H.Hong, White-box AES implementation revisited, in Journal of Communications and Networks, vol. 18(3), (2016), pp.273-287.

[18] R. L. Rivest, Cryptography and machine learning, in International Conference on the Theory and Application of Cryptology, (1991), pp.427-439.

[19] R. Bost, R. A. Popa, S. Tu, and S. Goldwasser, Machine learning classification over encrypted data, in NDSS, (2015).

[20] T. Graepel, L. Kristin, and M. Naehrig, ML confidential: Machine learning on encrypted data, in International Conference on Information Security and Cryptology,(2012), pp. 1-21.

[21] F. M. Barbosa, A.R.S.F. Vidal, H.L.S. Almeida, and F.L. DE Mello, Machine Learning Applied to the Recognition of Cryptographic Algorithms Used for Multimedia Encryption, in IEEE Latin America Transactions, vol. 15(7),(2017), pp. $1301-1305$

[22] H. Maghrebi, T.Portigliatti, And E. Prouff, Breaking cryptographic implementations using deep learning techniques, in International Conference on Security, Privacy, and Applied Cryptography Engineering, (2016), pp. 3-26.

[23] J.S. MUlLeR, Explicit Kummer surface formulas for arbitrary characteristic, in LMS Journal of Computation and Mathematics, vol. 13, (2010), pp. 47-64.

[24] A. Garbagnati, AND A. SARTI, Kummer surfaces and K3 surfaces with $\left(\frac{Z}{2 Z}\right)^{4}$ symplectic action, in Rocky Mountain Journal of Mathematics, vol. 46(4),(2016), pp. 1141-1205.

[25] Standard, AdVAnCe Encryption, Federal information processing standards publication 197, in FIPS PUB, (2001), pp. 46-53.

[26] C. Kumar, and M.N.Doja, A Novel Framework for Portfolio Selection Model Using Modified ANFIS and Fuzzy Sets, in Computers, vol. 7(4), (2018), pp. 57-64.

[27] R. K. Chahar, G. Datta, and N. Rajpal, Design of a new Security Protocol, in International Conference on Computational Intelligence and Multimedia Applications, vol. 4, (2007), pp. 132-136.

[28] N. Ferguson, K. John, L. Stefan, S. Bruce, S. Mike, W. David, and W. Doug, Improved cryptanalysis of Rijndael, in International Workshop on Fast Software Encryption, (2000), pp. 213-230.

[29] K. Deb, A.Pratap, S. Agarwal, and T. A. M. T. Meyarivan, A fast and elitist multiobjective genetic algorithm: NSGA-II, in IEEE transactions on evolutionary computation, vol. 6(2), (2002), pp. 182-197.

Edited by: Khaleel Ahmad

Received: Nov 25, 2018

Accepted: Feb 11, 2019 